\title{
AVALIAÇÃO DO PROGRAMA DE CONTROLE DA ESQUISTOSSOMOSE (PCE/PCDEN) EM MUNICÍPIOS SITUADOS NA BACIA DO RIO SÃO FRANCISCO, MINAS GERAIS, BRASIL
}

\author{
Maria Fernanda Lima e Costa, Henrique L. Guerra, Fabiano G. Pimenta \\ Junior, Josélia O.A. Firmo e Elizabeth Uchoa
}

Foi feita una avaliação do programa de controle da esquistossomose (PCE/PCDEN) na região da Bacia do Rio São Francisco em Minas Gerais. A área em estudo compreende seis municipios, com 130.000 habitantes e 916 localidades en uma área de $10.722 \mathrm{~km}^{2}$. As atividades tiveram início em quatro municipios entre 1983 e 1985 e em dois outros em 1987. As principais medidas de controle adotadas foram tratamentos sucessivos com oxamniquine e aplicações de niclosamida em coleções hídricas. A prevalência da infeç̧ão pelo Schistosoma mansoni nos primeiros quatro monicipios, que inicialmente estava entre 18 e $32 \%$, diminuin abruptamente após a primeira intervenção (1984/85) e permaneceu em niveis inferiores aos iniciais até a última avaliação realizada (1990/94); tendência semelhante foi observada para a proporçẫo de caranujos infectados. Nestes municipios, a proporção de localidades sem a infecção on com prevalências inferiores a $5 \%$ aumentou em detrimento daquelas com níveis mais altos de prevalência. Nos dois outros municipios, com prevalências iniciais inferiores a $5 \%$ não foram observadas mudanças substanciais nos indicadores endêmicos; a relação custo beneficio do programa nos últimos municípios deve ser avaliada e as prioridades redirecionadas para erradicar as áreas focais e prevenir a expansão para áreas indenes. Os autores chamam a atenção para as dificuldades a longo prazo de um programa de controle findamentado em tralamentos sucessivos. Informaçôes sobre os fatores determinantes da infeç̧ão pelo S. mansoni em cada localidade, ou em conjunto de localidades semelhantes, permitiriam a elaboração de mediulas complementares ao tratamento mais duradouras e menos dependentes da contínua uilização desie.

Palavras-chaves: Esquistossomose. Controle. Avaliação.

Programas para o controle da esquistossomose no Brasil, compreendendo grandes áreas de abrangência, foram desenvolvidos pelo Ministério da Saúde a partir de 1976. Neste ano foi implementado o Programa Especial de Controle da Esquistossomose (PECE) na região nordeste. As medidas adotadas incluíam tratamento com oxamniquine, muitas vezes em larga escala ou em

\footnotetext{
Laboratório de Epidemiologia e Antropologia Médica, Centro de Pesquisas René Rachou, Departamento de Medicina Preventiva e Social, Universisade Federal de Minas Gerais, MG e Fundaçäo Nacional de Saúde, Regional de Minas Gerais (MS/FNS), Belo Horizonte, MG.

Trabalho financiado pela Fundação Nacional de Saúde/ CODEPRO/Banco Mundial e pela Financiadora de Esludos e Projetos (FINEP).

Endereço para correspondência: Dra. Maria Fernanda Lima e Cosla. Laboratório de Epidemiologia e Antropologia Médica/ Centro de Pesquisas René Rachou. Caixa Postal 1743, 30161 970 Belo Horizonte, MG.

Recebido para publicaçāo em 16/12/95.
}

massa, ampla utilização de moluscicida e ações de educação e saneamento em áreas localizadas ${ }^{1219}$. A diversidade na metodologia adotada para a implementação do PECE em diferentes áreas endêmicas tornou difícil a sua avaliação. As primeiras tentativas de avaliação deste programa, efetuadas até 5 anos após a sua implantação, mostraram uma tendência na redução da prevalência da infecção pelo Schistosoma mansoni em diversos municípios do Rio Grande do Norte, Pernambuco e Paraiba ${ }^{12}$. Na Paraíba, entretanto, quando a análise foi feita porlocalidade, verificou-se a persistência da infecção em índices de prevalência iguais ou superiores a $20 \%$ em $47 \%$ daquelas situadas no litoral sul e em 11-14\% das localidades situadas em outras regiões do Estado². Admite-se também que o PECE tenha sido efetivo para a redução da morbidade da esquistossomose ${ }^{1119}$.

No Estado de Minas Gerais, a esquistossomose 
Lima e Costa MF, Guerra HL, Pimenta Junior FG, Firmo JOA, Uchoa E. Avaliação do Programa de Controle da Esquistossomose (PCE/PCDEN) em municípios siluados na Bacia do Rio São Francisco, Minas Gerais, Brasil. Revista da Sociedade Brasileira de Medicina Tropical 29:117-126, mar-abr, 1996.

mansoni ocorre predominantemente nas regiões norte e nordeste, estimando-se a sua prevalência em $10 \%$ da população escolar ${ }^{9}$. A região endêmica compreende 431 Municípios, correspondendo a uma área de $300 \mathrm{mil} / \mathrm{km}^{2}$, com cerca de 10 milhões de habitantes ${ }^{4}$. O Programa de Controle da Esquistossomose (PCE) neste Estado, desenvolvido pela Superintendência de Campanhas de Saúde Pública (SUCAM), teve início em 1983. Até esta época, atividades de controle haviam sido desenvolvidas pela SUCAM somente no município de Araxá, polo importante de turismo situado no extremo oeste do Estado. A partir de 1983 o programa se expande para o norte e nordeste de Minas Gerais: entre 1983 e 1986 para cinco municípios, entre 1987 e 1989 para mais oito municípios e a partir de 1990 para 141 municípios. Em 1989 o PCE é incluído nas atividades do Programa de Controle de Doenças Endêmicas (PCDEN) e em 1991 a SUCAM é incorporada à Fundação Nacional de Saúde. A partir de 1993, a área a ser coberta pelo programa é ampliada, através de convênios com municípios. As medidas de controle adotadas priorizam o tratamento com oxamniquine e atividades malacológicas. Somente em 1993 foram iniciadas obras de saneamento básico em áreas com prevalência da infecção superiores a $30 \%$. Atividades de educação para saúde, incluindo mobilização comunitária, foram incentivadas a partir de $1989^{4} 20$.

$O$ presente trabalho refere-se a uma avaliação preliminar do PCE/PCDEN entre 1983 e 1994 na região da Bacia do São Francisco, situada no norte do Estado de Minas Gerais. A região sob avaliação compreende seis municípios, com uma área de $10.722 \mathrm{~km}^{2}$, totalizando 132.565 habitantes ${ }^{7}$. O principal objetivo do estudo é descrever, a nível de localidade e/ou município, a evolução da prevalência da infecção pelo $S$. mansoni e da proporção de caramujos infectados após a implantação das medidas de controle e comparar estes indicadores ao observado antes da implantação destas medidas.

\section{MATERIAL E MÉTODOS}

\section{Área em estudo}

Foram incluídos na presente avaliação os municípios situados na Bacia do Rio São Francisco nos quais as atividades de controle da esquistossomose foram iniciadas entre 1983 e 1984 (Brasilia de Minas, Coração de Jesus, Mirabela e São João da Ponte) e aqueles pertencentes à mesma bacia hidrográfica cujas atividades tiveram início mais tarde (Engenheiro Navarro e Lagoa dos Patos) (Figura 1). O Município de Montes Claros, também situado na Bacia do São Francisco, foi excluído por ser o principal polo econômico da região e apresentar características muito diferentes dos demais. Também, foram excluídos três municípios situados na mesma Bacia Hidrográfica (Claro dos Poções, Ibiaí e Ubaí) porque a faixa etária alvo do programa diferiu nas três intervenções realizadas $(1987,1989$ e 1994). O PCE/PCDEN na área em estudo foi desenvolvido pela equipe do Distrito Sanitário de Montes Claros da Fundação Nacional de Saúde em Minas Gerais.

\section{Informações sobre o PCE/PCDEN}

As informações para o desenvolvimento do presente trabalho foram obtidas através da verificação dos arquivos existentes na sede do Distrito Sanitário em Montes Claros ou na sede regional da Fundação Nacional de Saúde em Belo Horizonte e através de entrevistas realizadas com membros da equipe técnica responsável pelo programa. Foram utilizadas as fichas adotadas na rotina do serviço, correspondentes ao resumo mensal de atividades de coproscopia por localidade e ao resumo mensal de atividades de malacologia por localidade, assim como uma ficha específica sobre saneamento, introduzida por decisão local do Distrito Sanitário de Montes Claros e utilizada somente na área coberta por este Distrito. Além disso, tendo por base dados correspondentes às atividades diárias de coproscopia e tratamento, foi construída uma nova ficha com informações sobre a faixa etária dos habitantes, faixa etária daqueles submetidos a coproscopia e o resultado deste exame. Esta ficha introduziu uma modificação na anotação da época em que as atividades de controle foram desenvolvidas, uma vez que não havia uniformidade na forma como esta informação era rotineiramente anotada. Todas as vêzes em que as atividades tiveram início em um ano mas só foram concluídas no ano seguinte, adotou-se este último para efeitos da análise. As seguintes informações foram consideradas: ano da atividade do programa, município, número de habitantes, número de 
Lima e Costa MF, Guerra HL, Pimenta Junior FG, Firmo JOA, Uchoa E. A valiação do Programa de Controle da Esquistossomose (PCE/PCDEN) en municipios situados na Bacia do Rio São Francisco, Minas Gerais, Brasil. Revista da Sociedade Brasileira de Medicina Tropical 29:117-126, mar-abr, 1996.

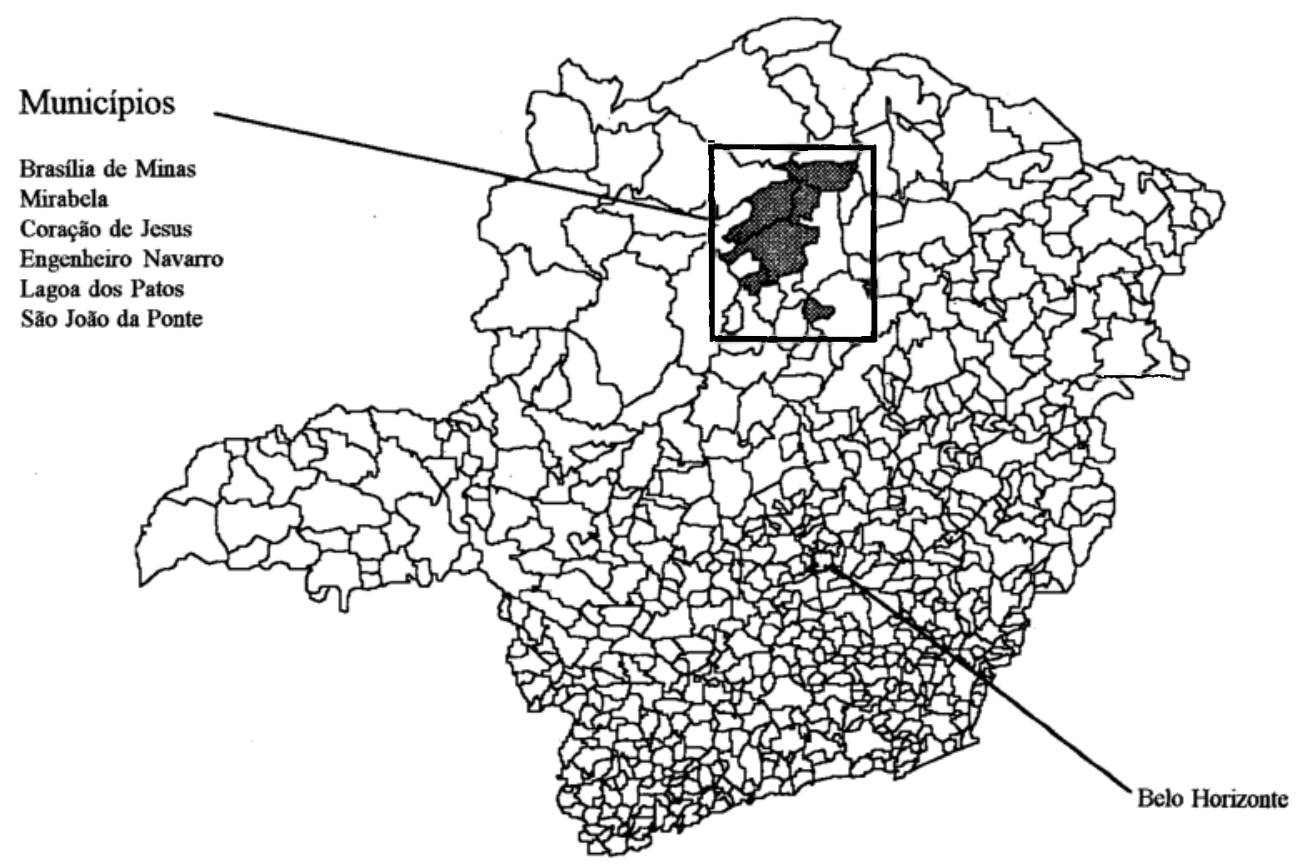

Figura 1 - Municípios selecionados para avaliação do Programa de Controle da Esquistossomose (PCE/PCDEN) na área da Bacia do São Francisco (Estado de Minas Gerais, 1983/1984).

domicílios, número de domicílios com água encanada, número de domicílios com esgoto, número de caramujos capturados, número de caramujos positivos para o $S$. mansoni, espécies existentes, quantidade de moluscicida utilizada, número de exames coproscópicos, número de pessoas elegíveis para o tratamento, número de pessoas tratadas e consumo de oxamniquine. Não foram considerados os dados sobre contagens de ovos de $S$. mansoni nas fezes, uma vez que a recuperação desta informação exigia a verificação de resultados de 487.237 exames de fezes, o que não foi viável por limitações de financiamento e tempo.

\section{Atividades do PCE/PCDEN na área em estudo}

As atividades do PCE/PCDEN incluíam identificação das localidades a serem trabalhadas, censo completo de cada localidade, identificação das coleções hídricas, levantamento malacológico, coproscopia (método de Kato-Katz ${ }^{8}$ ), tratamento específico (oxamniquine) e aplicação de moluscicida nas coleções hídricas (niclosamida). Em 1993, atividades de saneamento foram introduzidas pelo PCDEN em três localidades situadas nos municípios . de Mirabela e Coração de Jesus, através de convênios realizados com as respectivas prefeituras municipais ${ }^{18}$. Atividades de educação para a saúde tiveram início a partir de 1989. Neste ano foi desenvolvido um projeto piloto de mobilização comunitária em uma localidade (Patis) situada no município de Mirabela ${ }^{20}$. Uma avaliação dos efeitos da mobilização comunitária nesta área está sendo realizada pela nossa equipe e será objeto de uma outra publicação ${ }^{21}$. Desde o início do PCE/PCDEN, informações sobre a transmissão da esquistossomose e sua prevenção eram repassadas pelos agentes de campo (guardas), através de palestras ou informações, àqueles a serem submetidos ao tratamento específico ${ }^{17}$. 
Lima e Costa MF, Guerga HL, Pimenta Junior FG, Firno JOA, Uchoa E. Avaliação do Programa de Controle da Esquistossomose (PCE/PCDEN) en municipios siluados na Bacia do Rio São Francisco, Minas Gerais, Brasil. Revista da Sociedade Brasileira de Medicina Tropical 29:117-126, mar-abr, 1996.

Os critérios adotados para tratamento, utilização de moluscicida e elegibilidade para o exame de fezes mudaram ao longo do tempo ${ }^{4}$. No primeiro período (1983-86), quando a prevalência da localidade era inferior a $4 \%$, somente os indivíduos que eliminavam ovos de $S$. mansoni (positivos) eram tratados; nas localidades com prevalência entre 4 e $60 \%$, todos os indivíduos na faixa etária de 5-25 anos eram tratados independente do resultado do exame de fezes; quando a prevalência era superior a $60 \%$, todos os habitantes eram tratados; a utilização de moluscicida era feita sempre que caramujos infectados eram encontrados; o inquérito coproscópico era censitário, ou seja, todos os habitantes eram elegíveis para este exame. No segundo período (1987-89), quando a prevalência era inferior a $25 \%$, somente os positivos eram tratados; naquelas onde a prevalência variava entre 25 e $50 \%$ todos os habitantes na faixa etária de 714 anos eram tratados; naquelas com prevalência superior a $50 \%$ todos os habitantes eram tratados; a utilização de moluscicida se restringiu às coleções hídricas consideradas de importância epidemiológica (para definir a importância epidemiológica, pontos eram atribuílos às atividades da população na referida coleção, ao acesso à mesma, à velocidade de fluxo da água, à existência de caramujos, à contaminação - determinada pela presença ou não de vestígios de fezes, à utilização por pessoas infectadas e à colaboração da comunidade $\left.{ }^{16}\right)$; o inquérito coproscópico para as áreas nas quais as atividades começaram entre 1987 e 1988 foi restrito à população com 7-14 anos de idade; para aquelas localidades nas quais as atividades haviam se iniciado nas fases anteriores o inquérito permaneceu sendo censitário, por decisão local do Distrito Sanitário de Montes Claros, para permitir futuras avaliações (FG Pimenta Junior: comunicação pessoal). No terceiro período (após 1990), quando a prevalência era inferior ou igual a $50 \%$, somente os positivos eram tratados; naquuelas com prevalência superior a $50 \%$, todos os habitantes eram tratados; o inquérito coproscópico foi censitário e o levantamento malacológico não foi realizado nos municípios avaliados.

\section{RESULTADOS}

A Tabela 1 mostra o número de localidades expostas ao PCE/PCDEN, segundo o município, o ano e as atividades desenvolvidas. A área coberta pelo programa possuía em média 130.655 habitantes. Atividades foram desenvolvidasem 916 localidades e incluíram inquérito coproscópico, tratamento com oxamniquine, inquérito malacológico e aplicação de moluscicida. As atividades na área em estudo foram interrompidas entre 1991 e 1993 e retomadas em 1994, quando o levantamento malacológico não

Tabela 1 - Nímero de habitantes e de localidades expostas ao PCE/PCDEN, segundo o município, o ano e as atividades desenvolvidas (Bacia do Säo Francisco, Minas Gerais, 1983-94).

\begin{tabular}{|c|c|c|c|c|c|c|c|c|c|c|c|}
\hline \multirow[t]{2}{*}{ Município } & \multirow{2}{*}{$\begin{array}{c}\mathrm{N}^{0} \mathrm{de} \\
\text { habilantes }\end{array}$} & \multirow{2}{*}{$\begin{array}{c}\mathrm{N}^{\mathbf{O}} \mathrm{de} \\
\text { localidades }\end{array}$} & \multicolumn{9}{|c|}{ Número de localidades } \\
\hline & & & 1983 & 1984 & 1985 & 1986 & 1987 & 1988 & 1989 & 1990 & 1994 \\
\hline I Brasilia de Minas & 42.186 & 263 & & $200^{1,2,3,4}$ & $227^{1,2,3}$ & & $209^{1,2,3}$ & & $208^{1,2,3}$ & & \\
\hline II Coração de Jesus & 34.926 & 273 & $271^{2}$ & $271^{1,2,3,4}$ & $272^{1,2,3}$ & & $272^{1,2,3}$ & & $277^{1,2,3}$ & & \\
\hline III Mirabela & 15.604 & 97 & & $97^{1,2,3,4}$ & $97^{1,2,3}$ & & & $99^{1,2,3}$ & & $98^{1,2,3}$ & $96^{1}$ \\
\hline IV São João da Ponte & 29.829 & 203 & & & $205^{1,2,3,4}$ & $206^{1,2,3}$ & & $197^{1,2,3}$ & & & \\
\hline $\mathrm{V}$ Engenheiro Navarro & 5.195 & 44 & & & & & $44^{1,2,3,4}$ & & $43^{1,2,3}$ & & $45^{I}$ \\
\hline VI Lagoa dos Patos & 2.915 & 36 & & & & & $35^{1,2,3,4}$ & & $37^{1,2}$ & & $36^{1}$ \\
\hline
\end{tabular}

Total

130.655

916

\footnotetext{
a Número médio de habitantes e de localidades nos anos em que atividades do programa foram desenvolvidas (censo SUCAM/ FNS)

Inquérito coproscópico e tratamento com oxamiquine

2 Inquérito malacológico

3 Aplicação de mosluscicida

${ }^{4}$ Levantamento sobre condiçōes de saneamento

- Inquérito coproscópico na faixa etária de 7-14 anos
} 
Lima e Costa MF, Guerra HL, Pimenta Junior FG, Firmo JOA, Uchoa E. Avaliação do Programa de Controle da Esquistossomose (PCE/PCDEN) em municipios situados na Bacia do Rio São Francisco, Minas Gerais, Brasil. Revista da Sociedade Brasileira de Medicina Tropical 29:117-126, mar-abr, 1996.

foi mais realizado. As informações sobre saneamento básico restringiam-se ao período do início do PCE em cada município. As proporções de domicílios com água encanada eram 33,6, 8,3, 17,5 , 9,4, 49,3 e 25,2\%, respectivamente, em Brasília de Minas, Coração de Jesus, Mirabela, São João da Ponte, Engenheiro Navarro e Lagoa dos Patos. Ligações de esgoto existiam somente em Brasília de Minas (12,5\% dos domicílios) e Coração de Jesus $(2,1 \%)$.

A dimensão das atividades desenvolvidas pode ser verificada na Tabela 2. Entre 1983 e 1994 foram realizados 487.237 exames de fezes, foram realizados 179.351 tratamentos, foram utilizadas 330.961 cápsulas de oxamniquine e $430.844 \mathrm{ml}$ de xarope deste medicamento, foram capturados 225,489 caramujos e foram utilizados $221,9 \mathrm{~kg}$ de niclosamida. A cobertura das atividades foi alta: dos 535.715 indivíduos elegíveis para coproscopia entre 1984 e 1994, 487.237 (91,0\%) foram submetidos a exame de fezes; dos 225.489 caramujos capturados, $222.853(98,8 \%)$ foram examinados para classificação de espécie e infecção pelo $S$. mansoni; dos 213.106 indivíduos elegíveis para o tratamento segundo os critérios adotados nos diferentes períodos, $179.351(84,2 \%)$ foram tratados.

A Tabela 3 mostra o índice de infecção em caramujos (por mil) nos anos da realização do levantamento malacológico. Para Brasinia de Minas, Coração de Jesus, Mirabela e São João da Ponte observa-se um nítida redução da infecção após a primeira intervenção (1983-85); este padrão persistiu até 1989-90, época do último levantamento malacológico. Para os demais municípios (Engenheiro Navarro e Lagoa dos Patos), nos quais a infecção era inicialmente baixa, não foram observadas alterações importantes antes (1987-88) e após intervenção (1989-90). As espécies encontradas na área foram Biomphalaria glabrata, B. straminea $e$ B. tenagophia. Devido à forma como este dado é anotado nas fichas, não foi possível saber a distribuição das espécies na área em estudo; segundo os técnicos responsáveis, a B. glabrata é amplamente predominante (FG Pimenta Júnior; comunicação pessoal).

Tabela 2-Número de procedimentos realizados durante o PCE/PCDEN(Bacia do Säo Francisco, Minas Gerais, 198394.

\begin{tabular}{|c|c|c|c|c|c|c|}
\hline \multirow{2}{*}{ Município } & \multirow{2}{*}{$\begin{array}{l}\mathrm{N}^{\circ} \mathrm{de} \\
\text { coproscopias } \\
\text { realizadas }\end{array}$} & \multirow{2}{*}{$\begin{array}{l}\mathrm{N}^{0} \text { de } \\
\text { tratamentos } \\
\text { realizados }\end{array}$} & \multicolumn{2}{|c|}{ Consumo de oxamniquine } & \multirow{2}{*}{$\begin{array}{l}\text { No de } \\
\text { caramujos } \\
\text { capturados }\end{array}$} & \multirow{2}{*}{$\begin{array}{c}\text { Quant. de } \\
\text { moluscicida } \\
\text { empregada (kg) }\end{array}$} \\
\hline & & & capsulas (n) & xarope $(\mathrm{ml})$ & & \\
\hline I Brasílià de Minas & 155.724 & 58.947 & 107.794 & 151.712 & 64.417 & 64.417 \\
\hline Il Coração de Jesus & 159.296 & 61.720 & 140.903 & 118.543 & 81.748 & 81.748 \\
\hline III Mirabela & 73.846 & 27.305 & 46.466 & 54.954 & 22.123 & 22.123 \\
\hline IV São João da Ponte & 83.389 & 27.853 & 51.318 & 74.397 & 50.745 & 50.745 \\
\hline $\mathrm{V}$ Engenheiro Navarro & 9.439 & 2.628 & 5.340 & 6.138 & 3.224 & 3.224 \\
\hline VI Lagoa dos Patos & 5.503 & 898 & 1.500 & 2.740 & 3.196 & 3.196 \\
\hline Total & 487.237 & 179.351 & 330.961 & 430.844 & 225.489 & 225.489 \\
\hline
\end{tabular}

Tabela 3 - Proporção de caranujos infectados em municípios expostos ao PCE/PCDEN em anos da realização do levantamento malacológico (Bacia do São Francisco, Minas Gerais, 1983-90).

\begin{tabular}{lccccc}
\hline & \multicolumn{5}{c}{ Anos } \\
\cline { 2 - 5 } Município & $\begin{array}{c}1983-84 \\
\%\end{array}$ & $\begin{array}{c}1985 \\
\%\end{array}$ & $\begin{array}{c}1986 \\
\%\end{array}$ & $\begin{array}{c}1987-88 \\
\%\end{array}$ & $\begin{array}{c}\text { \% } \\
\end{array}$ \\
\hline I Brasília de Minas & 79,1 & 5,6 & - & 0,4 & 5,4 \\
Il Coração de Jesus & 38,8 & 3,9 & - & 1,2 & 4,0 \\
III Mirabela & 58,5 & 7,0 & - & 4,1 & 7,1 \\
IV São João da Ponte & - & 61,5 & 0,7 & 1,2 & - \\
V Engenheiro Navarro & - & - & & 1,0 & 1,7 \\
VI Lagoa dos Patos & - & - & & 2,1 & 2,6 \\
\hline
\end{tabular}


Lima e Costa MF, Guerra HL, Pimenta Junior FG, Firmo JOA, Uchoa E. Avaliação do Programa de Controle da Esquistossomose (PCE/PCDEN) em municipios situados na Bacia do Rio São Francisco, Minas Gerais, Brasil. Revista da Sociedade Brasileira de Medicina Tropical 29:117-126, mar-abr, 1996.

A Tabela 4 mostra a prevalência da infecção pelo $S$. mansoni em anos da realização do inquérito coproscópico. A prevalência da infecção nos municípios de Brasilia de Minas, Coração de Jesus, Mirabela e São João da Ponte, que inicialmente estava entre 18 e $32 \%$, diminuiu abruptamente após a primeira intervenção e permaneceu em níveis inferiores a $15 \%$ até 1989-90; em 1994, o inquérito foi realizado somente no município de Mirabela, verificando-se um aumento da prevalência da infecção para para 19,3\%. As mesmas tendências foram observadas quando foi considerada a mediana da prevalência por município (Tabela 5). Em Engenheiro Navarro e Lagoa dos Patos, a primeira determinação da prevalência (1987-88) foi feita somente na faixa etária de 7-14 anos; em ambos os municípios, a prevalência da infecção nesta faixa etária era inferior $\mathrm{a} 5 \%$. Nos dois periodos seguintes (1987-88 e 1994), quando o inquérito foi censitário, a prevalência da infecção no primeiro município passou de 2,2 para $6,9 \%$ e no segundo, de 0,2 para $1,5 \%$ (Tabela 4). A mediana da prevalência foi $0,0 \%$ em todos os períodos, independente de o inquérito ter sido censitário ou restrito à faixa de 014 anos de idade (Tabela 5).

A Figura 2 mostra a distribuição proporcional da prevalência da infecção pelo $S$. mansoni nas localidades situadas nos municípios comprevalências iniciais superiores a $15 \%$. A proporção de localidades sem a infecção ou com prevalências inferiores a $5 \%$ aumentou em todos os municípios durante o periodo considerado em detrimento daquelas com níveis mais altos de prevalência. Entretanto, na última avaliação realizada em cada município, existiam $14,4,14,4,49,0$ e $10,2 \%$ de localidades com prevalências acima de $15 \%$ em Brasília de Minas, Coração de Jesus, Mirabela e São João da Ponte, respectivamente.

A Figura 3 mostra a distribuição proporcional da prevalência da infecção nas localidades situadas nos municípios com prevalências iniciais em escolares inferiores a $5 \%$. Entre a segunda (198990) e a última intervenção (1994) diminuíram as proporções de localidades sem infecção pelo $S$. mansoni e aumentaram aquelas com prevalências entre $0,1-5,0 \mathrm{e}>5 \%$.

Tabela 4 - Prevalência da infecção pelo Schistosoma mansoni em municípios expostos ao PCE/PCDEN em anos da realização do inquérito coproscópico (Bacia do São Francisco. Minas Gerais, 1983-90).

\begin{tabular}{lcccccc}
\hline & \multicolumn{6}{c}{ Anos } \\
\cline { 2 - 7 } Município & 1984 & 1985 & 1986 & $1987-88$ & $1989-90$ & 1994 \\
& $\%$ & $\%$ & $\%$ & $\%$ & $\%$ & $\%$ \\
\hline I Brasília de Minas & 18,2 & 8,0 & - & 5,7 & 7,4 & - \\
II Corąão de Jesus & 33,2 & 9,9 & - & 8,2 & 8,9 & - \\
III Mirabela & 30,1 & 11,5 & - & 13,9 & 12,4 & 19,3 \\
IV São João da Ponte & - & 18,8 & 5,9 & 6,4 & - & - \\
V Engenheiro Navarro & - & - & - & $4,2 *$ & 2,2 & 6,9 \\
VI Lagoa dos Patos & - & - & - & $3,0 *$ & 0,2 & 1,5 \\
\hline
\end{tabular}

* somente entre 7-14 anos de idade

Tabela S - Mediana da prevalência da infecção pelo Schistosoma mansoni em municipios expostos ao PCE/PCDEN em anos da realização do inquérito coproscópico (Bacia do São Francisco, Minas Gerais, 1983-90).

\begin{tabular}{lrrrrrr}
\hline & \multicolumn{7}{c}{ Anos } \\
\cline { 2 - 7 } Município & $\begin{array}{c}1984 \\
\%\end{array}$ & $\begin{array}{c}1985 \\
\%\end{array}$ & $\begin{array}{c}1986 \\
\%\end{array}$ & $\begin{array}{c}1987-88 \\
\%\end{array}$ & $\begin{array}{c}1989-90 \\
\%\end{array}$ & $\begin{array}{c}1994 \\
\%\end{array}$ \\
\hline I Brasília de Minas & 15,7 & 6,0 & - & 3,2 & 3,5 & - \\
II Coração de Jesus & 24,2 & 9,6 & - & 5,0 & 5,0 & - \\
III Mirabela & 27,3 & 10,0 & - & 10,3 & 9,6 & 15,1 \\
IV São João da Ponte & - & 9,1 & 2,8 & 2,1 & - & - \\
V Engenheiro Navarro & - & - & - & $0,0 *$ & $\mathbf{0 , 0}$ & 0,0 \\
VI Lagoa dos Patos & - & - & - & $0,0 *$ & $\mathbf{0 , 0}$ & 0,0 \\
\hline
\end{tabular}

* somente entre 7-14 anos de idade 
Lima e Costa MF, Guerra HL, Pimenta Junior FG, Firmo JOA, Uchoa E. Avaliação do Programa de Controle da Esquistossomose (PCE/PCDEN) em municípios situados na Bacia do Rio São Francisco, Minas Gerais, Brasil. Revista da Sociedade Brasileira de Medicina Tropical 29:117-126, mar-abr, 1996.
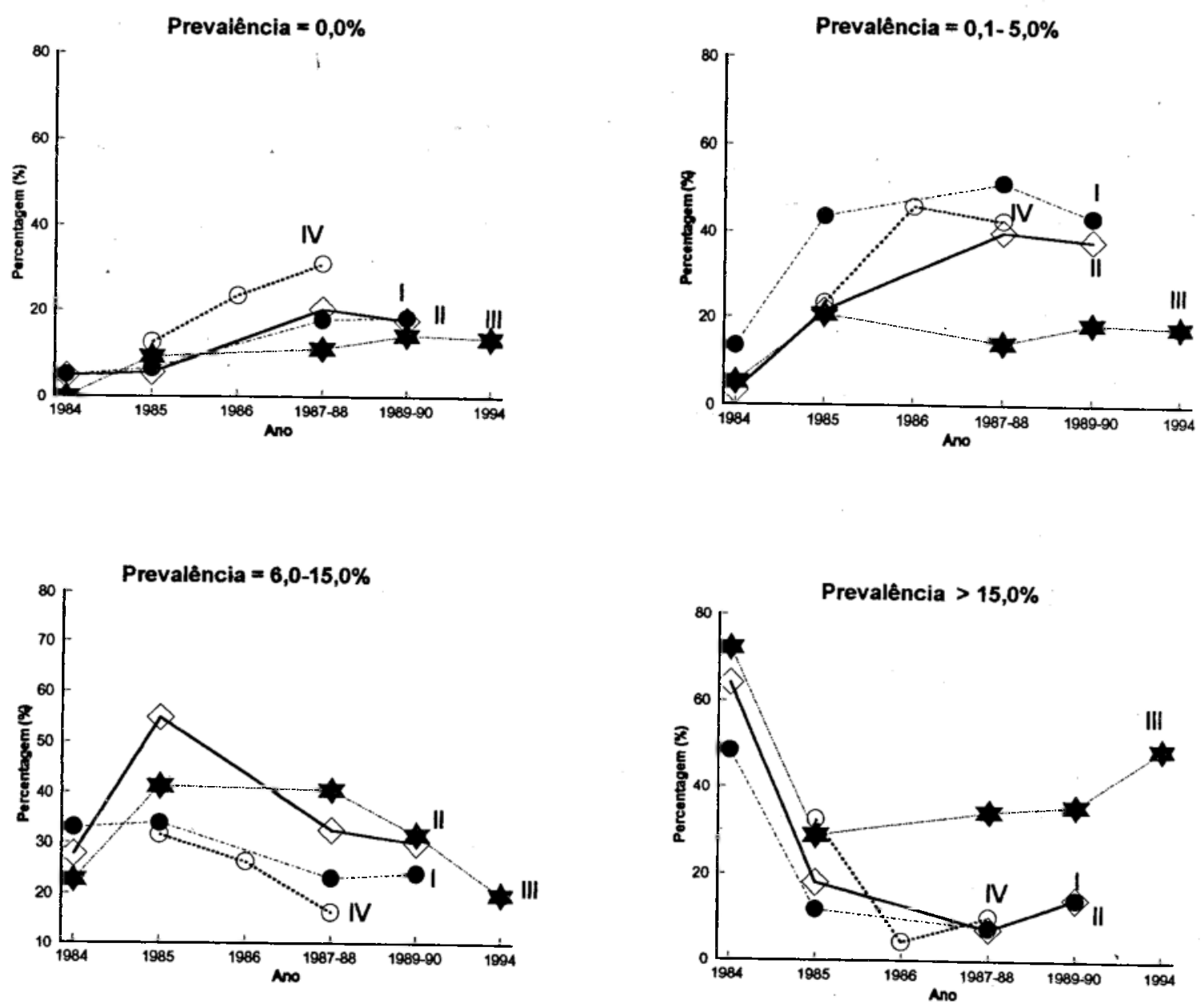

\% em relação ao total de localidades em cada Município

Figura 2 - Distribuição proporcional da prevalência da infecção pelo Schistosoma mansoni em localidades situadas em Municípios com prevalências iniciais superiores a 15\%, segundo o ano: I-Brasilia de Minas, II - Coração de Jesus, III - Mirabela e IV - São João da Ponte (PCE/PCDEN, Bacia do São Francisco, Minas Gerais, 1984-94).
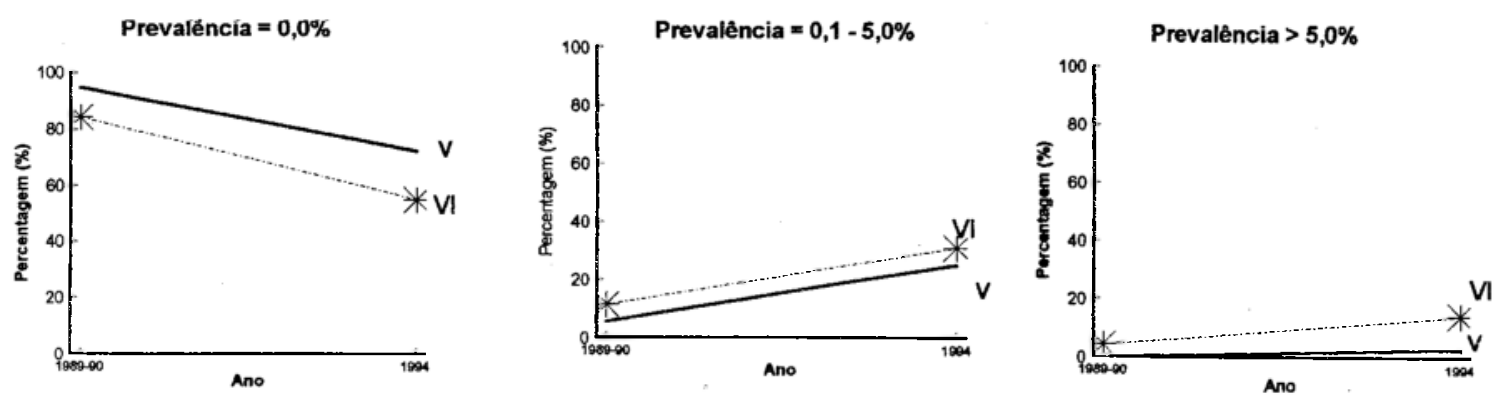

\% em relação ao total de localidades em cada Município

Figura 3 - Distribuição proporcional da prevalência da infecção pelo Schistosoma mansoni em localidades situadas em Municípios com prevalências iniciais superiores a 5\%, segundo o ano: V-Engenheiro Navarro e VI - Claro dos Poções (PCE/PCDEN, Bacia do São Francisco, Minas Gerais, 1989-94). 
Lina e Costa MF, Guerra HL, Pimenta Junior FG, Firmo JOA, Uchoa E. Avaliação do Programa de Controle da Esquistossomose (PCE/PCDEN) em municípios siluados na Bacia do Rio São Francisco, Minas Gerais, Brasil. Revista da Sociedade Brasileira de Medicina Tropical 29:117-126, mar-abr, 1996.

\section{DISCUSSÃO}

Foram verificadas duas tendências na prevalência da infecção pelo $S$. mansoni e no índice de infeç̧ão em caramujos na região estudada. No primeiro conjunto de municípios, caracterizado por prevalências inicias mais altas, observou-se uma nítida redução deste indicadores após o início do programa de controle da esquistossomose, que persistiu até a última avaliação realizada. No segundo conjunto de municípios. caracterizado por prevalências iniciais mais baixas e por grande proporção de localidades sem a infecção, não foram observadas mudanças substanciais naqueles indicadores, verificando-se uma discreta tendência à elevação da prevalência da infecção na última avaliação realizada.

Uma das maiores dificuldades para a avaliação de Programas de Controle da Esquistossomose em áreas extensas está relacionada ao caráter focal da endemia $^{22}$. A análise dos dados agregados por município pode produzir importantes vieses. A análise de indicadores por localidade, da mesma forma, está sujeita a distorções por instabilidade devido a pequenos números; na área estudada, $37,4 \%$ das localidades possuíam menos de 50 habitantes. Para tentar atenuar este problema, nós utilizamos a mediana da prevalência em cada município, além da prevalência global. Além disso, quando a análise da distribuição da prevalência proporcional por localidade foi feita, nós inicialmente estratificamos os dados segundo o número de habitantes por localidade $(1-49 \mathrm{e}>50)$. Como as tendências observadas foram semelhantes, a análise final foi apresentada, desconsiderando a estratificação. Uma dificuldade adicional referiu-se a mudanças nas faixas etárias alvo do programa nos municípios nos quais as atividades de controle tiveram início a partir de 1987. Em três municípios (Claro dos Poções, Ibiaí e Ubaí) foram inicialmente examinados indivíduos com 0-25 anos (em 1987) e sucessivamente aqueles com 7-14 anos e indivíduos de todas as faixas etárias (em 1989 e 1994, respectivamente). Isto levou à exclusão destes municípios para evitar erros de interpretação das tendências observadas, uma vez que não se referiam à mesma população.
Por outro lado, a existência de informações detalhadas das atividades do programa em Minas Gerais permitiu examinar e descartar potenciais fontes de tendenciosidade relacionadas à validade interna: 91,0\% dos habitantes da área coberta pelo programa foram submetidos ao exame de fezes, $84,2 \%$ dos indivíduos elegíveis foram tratados com oxamniquine e foram examinados $98,8 \%$ dos caramujos capturados.

Estudos conduzidos em diferentes áreas endêmicas em Minas Gerais descreveram dois padrões para a predição da infecção pelo $S$. mansoni. Em áreas onde o abastecimento de água era precário, a infecção e/ou as esplenomegalias estavam associadas à ausência de abastecimento de água no domicílio e aos contatos com águas para lazer ${ }^{12} 15$. Em outras, onde existia maior proporção de domicílios com água encanada ou cisterna, a infecção estava associada somente ao lazer e/ou ao trabalho na lavoura ${ }^{5613}$. Não existem informações sobre a área em estudo que permitam verificar a infuência destes padrões sobre a evolução da endemia. De qualquer forma, a queda abrupta da prevalência, como verificada no primeiro conjunto de municípios, é geralmente conseqüência do tratamento específico. A sucessão de tratamentos em pequenos intervalos de tempo é também efetiva para manter baixa a prevalência da infecção pelo $S$. mansoni ${ }^{6}{ }^{10}$. Entretanto, a reinfecção ocorre e a sua incidência aumenta à medida em que aumentam os intervalos entre os tratamentos ${ }^{3}{ }^{14}$. No presente trabalho, verificou-se uma tendência àelevação da prevalência da infecção no município de Mirabela (de $12 \% \mathrm{em}$ 1990 para $19 \%$ em 1994), quando o tratamento foi interrompido por três anos consecutivos.

Os objetivos do Programa de Controle da Esquistossomose dentro do PCDEN são os seguintes: a) suprimir as formas graves pela redução da prevalência da infecção pelo $S$. mansoni; b) reduzir a prevalência da infecção a níveis inferiores a $5 \%$; c) erradicar as áreas focais e d) prevenir a expansão para áreas vulneráveis indenes ${ }^{4}$. Tendo em vista estes objetivos, nossos resultados levam às seguintes conclusões:

1) não foi possível avaliar o impacto do PCE/ PCDEN na evolução da morbidade da esquistossomose na área em estudo, uma vez que 
Lima e Costa MF, Guerra HL, Pimenta Junior FG, Firmo JOA, Uchoa E. Avaliação do Programa de Controle da Esquistossomose (PCE/PCDEN) em municípios situados na Bacia do Rio São Francisco, Minas Gerais, Brasil. Revista da Sociedade Brasileira de Medicina Tropical 29:117-126, mar-abr, 1996.

não existiam dados anteriores e posteriores à intervenção sobre a condição clínica dos habitantes, o que seria inviável pela extensão do programa;

2) no que se refere aos municípios com prevalências iniciais da infeção pelo $S$. mansoni superiores a $15 \%$, houve nítida redução da prevalência da infecção, redução dos índices de infecção em caramujos e aumentaram as localidades nas quais a infecção não foi mais encontrada; entretanto, na última avaliação realizada, a meta ambiciosa de reduzir as prevalências a níveis inferiores a $5 \%$ não foi alcançada em 38, 44, 69 e $26 \%$ das localidades situadas, nos Municípios de Brasília de Minas, Coração de Jesus, Mirabela e São João da Ponte, respectivamente;

3) no que se refere aos Municípios de Engenheiro Navarro e Lagoa dos Patos, com prevalências iniciais em escolares inferiores a $5 \%$ e onde a infecção não foi encontrada na maioria das localidades, os efeitos do programa não foram visíveis; a relação custo benefício do programa nestes municípios deve ser avaliada e as atividades redirecionadas para alcançar os objetivos c e d (erradicar as áreas focais e prevenir a expansão para áreas indenes), pelo menos nas localidades com prevalências inferiores a $5 \%$;

4) durante o início do PCE/PCDEN, a cobertura de abastecimento de água e de ligações de esgoto era precária; nos municípios que faziam parte da área em estudo, a proporção de domicílios com água encanada variava entre 8 e $49 \%$ e somente dois municípios possuíam ligações de esgoto (em $13 \mathrm{e}$ $2 \%$ dos domicílios, respectivamente); as atividades do Programa de Controle da Esquistossomose não incluíram melhorias do saneamento básico, exceto em três localidades em 1993.

A efetividade da quimioterapia para a redução da prevalência da infeç̧ão pelo $S$. mansoni e da sua morbidade é amplamente reconhecida 12310111422 . Entretanto, ainda não se sabe por quanto tempo estes benefícios persistem após a interrupção do tratamento. Um programa tendo por base tratamentos sucessivos de populações em áreas endêmicas pode ser inviável a longo prazo devido ao seu alto custo. Informações sobre os fatores determinantes da infecção pelo $S$. mansoni em cada localidade, ou em conjuntos semelhantes de localidades, permitiriam a elaboração de medidas complementares ao tratamento mais duradouras e menos dependentes da contínua utilização deste.

\section{SUMMARY}

An evaluation of the control program on schistosomiasis (PCE/PCDEN) was performed in the region of Säo Francisco river in Minas Gerais. The study area comprises six municipalities, with 130,000 inhabitants and 916 localities situated in an area with $10,722 \mathrm{~km}^{2}$. The activities initiated in 1983-85 in four municipalities and in 1987 in the other two. The main measures of control were repeated treatment with oxamniquine and use of niclosamide. The prevalence of infection by Schistosoma mansoni in the first four municipalities, that was initially around 18 and $32 \%$, dropped abruptly after the first intervention (1983/85) and remained in levels below the initial ones until the last assessment (1990-94); similar trends were observed for the proportion of infected snails. In these municipalities, the proportion of localities withou infection or with prevalence below $5 \%$ increased in relation to those with higher prevalence. In the other two municipalities, with initial prevalence below $5 \%$, there were no substantial changes in $\mathrm{S}$. mansoni prevalence or proportion of infected snails; the cost benefit of the program in these municipalities need to be assessed and the priorities redirected to eradicate focal areas and prevent spread to non infected localities. The authors call attention to the difficulties in the long term of a control program based on repeated treatments. Information on factors associated with S. mansoni infection in each locality, or in groups of similar localities, would allow to develop additional measures to treatment that cold last longer and be less dependent on the continuous use of chemotherapy.

Key-words: Schistosomiasis. Control. Evaluation.

\section{AGRADECIMENTOS}

Esta avaliação preliminar do PCE/PCDEN no norte de Minas Gerais foi possível pela existência de um bom arquivo no Distrito Sanitário de Montes Claros (MS-FNS), acessivel e em condições de utilização. Os autores agradecem à Dra. Lídia Sanguinetti de Souza Rabelo, Chefe do Distrito Sanitário de Montes Claros, e à equipe do setor técnico deste Distrito, sem cuja colaboração este trabalho não seria viável. Os autores agradecem também ao Dr. Roberto Sena Rocha pela revisão crítica do manuscrito e valiosas sugestões. 
Lima e Costa MF, Guerra HL, Pinenta Junior FG, Firmo JOA, Uchoa E. Avaliação do Programa de Controle da Esquistossomose (PCE/PCDEN) en municipios situados na Bacia do Rio São Francisco, Minas Gerais, Brasil. Revista da Sociedade Brasileira de Medicina Tropical 29:117-126, mar-abr, 1996.

\section{REFERÊNCIAS BIBLIOGRÁFICAS}

1. Almeida Machado P. The Brazilian Program for Schistosomiasis Control. American Journal of Tropical Medicine and Hygiene $31: 76-86,1982$.

2. Coura JR, Mendonça MZG, Madruga JP. Tentativa de avaliação do Programa Especial de Controle da Esquistossomose (PECE) no Estado da Paraíba, Brasil. Revista da Sociedade Brasileira de Medicina Tropical 20:67-76, 1987.

3. Coura Filho P, Rocha RS, Lima e Costa MFF, Kaiz N. A municipal level approach to the management of schistosomiasis control in Peri-Peri, MG, Brazil. Revista do Instituto de Medicina Tropical de São Paulo 4:543-548, 1992.

4. Drumond SC. Programa de controle da esquistossomose mansoni no Estado de Minas Gerais. Resumo de Avaliaçāo das Atividades do Projeto de Controle das Doenças Endemicas do Estado de Minas Gerais (1989-1994), Ministério da Saúde, Fundação Nacional de Saúde, Regional de Minas Gerais p.78-80. 1994.

5. Guerra HL. Epidemiologia da esquistossomose mansoni em uma área de média endemicidade em Minas Gerais. Tese, Universidade Federal de Minas Gerais, 1992.

6. Firmo JA. Avaliação clínico-epidemiológica da esquistossomose mansoni em uma área da periferia de Belo Horizonte. Tese, Universidade Federal de Minas Gerais, 1994.

7. IBGE. Fundação Instituto Brasileiro de Estatística. Resultados Preliminares do Censo Demográfico de Minas Gerais, (mimeo)/IBGE - CEI/FJP, 1991.

8. Katz N, Chaves A, Pellegrino JP. A simple device for quantitative stool thick-smear technique in schistosomiasis mansoni. Revista do Instituto de Medicina Tropical de São Paulo 14:397-400, 1972

9. Katz N, Motta E, Oliveira VB, Carvalho EF. Prevalência da esquistossomose em escolares no Estado de Minas Gerais. In: Resumos do XVI Congresso da Sociedade Brasileira de Medicina Tropical, João Pessoa, p.102, 1978.

10. Katz N. Experiencias com quimiolerapia em grande escala no controle da esquistossomose no Brasil. Revista do Instituto de Medicina Tropical de São Paulo 22:40-51, 1980 .

11. Kloetzel K, Schuster NH. Repeated mass treatment of schistosomiasis mansoni: experience in hyperendemic areas of Brazil. 1. Parasilological effects and morbidity. Transactions of the Royal Society of Tropical Medicine and Hygiene $81: 365-370.1987$.

12. Lima e Costa MFF, Magalhães MHA, Rocha RS, Antunes
CMF, Katz N. Water contact patterns and socio-economic variables in the epidemiology of schistosomiasis mansoni in an endemic area in Brazil. Bulletin of the World Health Organization 65:57-66,1987.

13. Lima e Costa MFF, Rocha RS, Leite MLC, Carneiro RG, Colley D, Gazzinelli G, Katz N. A multivariate analysis of sociodemographic factors, water contact patterns and Schistosoma mansoni infection in an endemic area in Brazil. Revista do Instituto de Medicina Tropical de São Paulo $33: 58-63,1991$.

14. Lima e Costa MFF, Rocha RS, Coura Filho P, Katz N. A 13 year follow-up of treatment and snail control in an endemic area for Schistosoma mansoni in Brazil: incidence of infection and reinfection. Bulletin of the World Health Organization 71:197-205, 1993.

15. Lima e Costa MFF, Rocha RS, Magathäes MHA, Katz N. Um modelo hierárquico de análise das variáveis sócio econômicas e dos padrōes de contatos com águas associados à forma hepatoesplênica da esquistossomose. Cadernos de Saúde Pública 10(supl.2):241-253, 1994.

16. Ministério da Saúde. Fundação Nacional de Saúde. Registro para determinaçāo do potencial de transmissão das coleções hídricas (Tabela), 1987.

17. Ministério da Saúde. Fundação Nacional de Saúde. Manual do guarda de esquistossomose: instruçōes para coproscopia/ medicação, Brasilia, 1988.

18. Ministério da Saúde. Fundaçāo Nacional de Saúde. Regional de Minas Gerais. Cronograma Físico Financeiro e Plano de Aplicação (Tabełas) $n^{\circ} 01$ e $02,1993$.

19. Silveira AC. Controle da esquistossomose no Brasil. Memórias do Instituto Oswaldo Cruz 84 (suppl. I):91, 1989.

20. Thuin MMB. Mobilização comunitária - FNS. Programa de Controle da Esquistossomose Mansoni no Estado de Minas Gerais. Resumo de Avaliação das Atividades do Projeto de Controle das Doenças Endemicas do Estado de Minas Gerais (1989-1994), Ministério da Saúde, Fundação Nacional de Saúde, Regional de Minas Gerais p.133-135, 1994.

21. Uchoa ME, Lima e Costa MFF. Projeto de pesquisa para avaliação do impatio de programas IEC/MC no controle das doenças endemicas em cinco Estados do Nordeste: sub-projeto Minas Gerais. Ministério da Saúde, Fundação Nacional de Saúde, CODEPRO, Banco Mundial/PNUD, 1994.

22. World Health Organization. The cont rol of schistosomiasis. WHO Technical Report series No. 830, Geneve, 1994. 